\title{
ORIGINAL ARTICLE \\ Cervical spine injuries and collar complications in severely injured paediatric trauma patients
}

\author{
M Chan ${ }^{1}$, W Al-Buali ${ }^{2}$, T Charyk Stewart ${ }^{3}$, RN Singh ${ }^{4,5}$, A Kornecki ${ }^{4,5}$, JA Seabrook ${ }^{4,5}$ and DD Fraser ${ }^{4,5,6,7,8}$
}

Study design: A retrospective registry review.

Objectives: To determine the incidence of cervical spine (CS) injuries and collar complications in severely injured paediatric trauma patients.

Setting: Regional Trauma Centre, Children's Hospital.

Methods: A retrospective review of 365 paediatric severe trauma patients (0-17 years), defined as an Injury Severity Score $($ ISS) $\geqslant 12$, admitted to the paediatric intensive care unit (PICU).

Results: Clinically significant CS injuries occurred in $5 \%(n=18 / 365)$ of trauma patients, in $9 \%(n=13 / 149)$ of traumatic brain injury (TBI) patients and in 11\% $(n=6 / 56)$ of in-hospital trauma deaths. CS injuries were suspected before imaging in 33\% $(n=6 /$ 18) of patients based on either motor/sensory impairment or shock. CS injuries were deemed unstable in $61 \%(n=11 / 18)$ of patients. Patients with CS injuries had higher ISS, and longer PICU and hospital stays $(P<0.05)$. CS collar complications occurred in $10 \%$ of patients, mainly identified by day 6 and consisting of either erythema or ulcers. Patients with CS collar complications were older and more likely to have TBI, lower Glasgow Coma Scale (GCS) scores, longer PICU and hospital stays, and increased days to CS clearance $(P<0.05)$. Three CS $X$-rays, together with flexion/extension views, were used most frequently for CS clearance.

Conclusion: CS injuries were prevalent in severely injured paediatric trauma patients, particularly in those with TBI and in nonsurvivors. CS collar complications were associated with a lower GCS and longer CS clearance times. Attention to CS collar management protocols and earlier CS clearance with computed tomography/magnetic resonance imaging in obtunded patients might reduce CS collar complications.

Spinal Cord (2013) 51, 360-364; doi:10.1038/sc.2013.6; published online 5 March 2013

Keywords: children; severe trauma; cervical spine; injury; clearance; collar complications

\section{INTRODUCTION}

Paediatric cervical spinal (CS) injuries, defined as boney, soft tissue and/or spinal cord injury in the cervical spine region, can result in severe morbidity and mortality. ${ }^{1}$ The incidence of CS injuries in the general paediatric trauma population has been reported at $\sim 0.2-$ $1.6 \% .^{1-5}$ Children are at increased risk for cervical injury due to their underdeveloped neck muscles, proportionally larger heads, higher fulcrum (C2-C3 in children vs. C5-C6 in adults), incomplete ossification of the physes and apophyses, anterior wedging of the vertebra, joint capsule laxity and horizontally angulated facet joints. ${ }^{4,6,7}$

Severely injured paediatric trauma patients are often obtunded and/or ventilated. Clinical CS examination in paediatric severe trauma patients is frequently unreliable, and their neurological exam is often complicated by traumatic brain injury (TBI) and/or sedative medications. ${ }^{1,8}$ Early CS clearance is important to avoid CS collar complications and facilitate optimal patient care. In adults, the prevalence of skin breakdown and ulcers with prolonged use of CS collars ranges from 7 to $23 \%,{ }^{9,10}$ and CS collar complications are associated with increased length of stay, greater morbidity and increased costs of care. ${ }^{11,12}$

Paediatric severe trauma patients are among those with the greatest risk of both CS injuries and prolonged use of CS collars. The primary objectives of this study were to report the incidence of CS injuries and collar complications in this severely injured, vulnerable population.

\section{MATERIALS AND METHODS}

This study was approved by the Institutional Research Board at Western University. We performed a 10-year retrospective analysis of all severely injured paediatric (1 month to 17 years) trauma patients admitted to our regional paediatric trauma facility at Children's Hospital, London Health Sciences Centre (London, ON, Canada). Our trauma referral region spans $29,000 \mathrm{~km}^{2}$ with an estimated paediatric population ( $<18$ years of age) at 600000 .

Trauma patients were identified by manual searches of the paediatric intensive care unit (PICU) admission logbooks, and by computerized searches via the hospital records department for the years 1996-2005. Patient capture was verified with the Trauma Registry database, a provincially-mandated and professionally managed database that underwent regular data quality

${ }^{1}$ Paediatrics, University of British Columbia, Vancouver, British Columbia, Canada; ${ }^{2}$ Paediatrics and Critical Care Medicine, King Faisal University, Khobar, Saudi Arabia; ${ }^{3}$ Surgery, Western University, London, Ontaria, Canada; ${ }^{4}$ Paediatrics and Critical Care Medicine, Western University, London, Ontaria, Canada; ${ }^{5} \mathrm{Children's} \mathrm{Health} \mathrm{Research} \mathrm{Institute,}$ London, Ontaria, Canada; ${ }^{6}$ Centre for Critical Illness Research, London, Ontaria, Canada; ${ }^{7}$ Physiology/Pharmacology, Western University, London, Ontaria, Canada and ${ }^{8}$ Clinical Neurological Sciences, Western University, London, Ontaria, Canada

Correspondence: Dr DD Fraser, Paediatric Critical Care Medicine Office, Room C2-C82, Children's Hospital, London Health Sciences Center, 800 Commissioners Road East, London, Ontaria, Canada N6C 2V5.

E-mail: douglas.fraser@lhsc.on.ca

Received 6 November 2012; revised 12 January 2013; accepted 20 January 2013; published online 5 March 2013 
evaluation, with over 400 data points per trauma patient. Admission to our 12 bed medical/surgical PICU was based on severity of injuries, ventilation requirements, hemodynamic instability, post-operative recovery and/or need for continuous monitoring (that is, grade IV-V splenic/hepatic lacerations). Patients that died before CS imaging or without postmortem examination, as well as birth traumas, penetrating injuries, burns and Injury Severity Score (ISS) $<12$ were excluded.

Demographic, clinical and injury information abstracted from the charts included the patient's age, sex, primary injury, PICU and hospital length of stay days, ISS, associated injuries, initial Glasgow Coma Scale (GCS) score, radiological findings of spine and brain injuries, days from arrival to CS clearance, method(s) of CS clearance and CS collar complications. The primary injury was defined as either the only injury or the injury most likely to result in severe morbidity or mortality. For those patients with CS collar complications, the data recorded included the PICU day the complication was first noted, type of complication (erythema or ulcer/pressure sore, other), location of the complication and what type of collar the child was wearing.

Severe TBI was defined as GCS $\leqslant 8$ with abnormalities noted on admission head computed tomography (CT), including cerebral oedema, diffuse axonal injury, contusion, haemorrhage, herniation and/or midline shift.

Clinically significant CS injuries were defined as any abnormality on CS imaging, including fractures, dislocations, subluxations, distractions, disk herniations and/or ligamentous injury. Spinal stability was defined as the ability of the spine under physiological loads to limit patterns of displacement, so as to not damage or irritate the cord and/or nerve roots. Conversely, spinal instability refers to excessive displacement of the spine that would result in neurological deficit, deformity or pain. Spinal instability resulted in stabilization, either external (rigid CS collar or Halo vest) or internal (fusion).

One- or Two-Piece Extrication Collars with age-appropriate color-coding were immediately applied to the trauma patients in the field by Emergency Medical Services. On arrival to our Regional Level-1 Trauma centre, CS collars were frequently changed to Philadelphia, Aspen or Miami brands to optimize support and comfort. CS collars were checked regularly for appropriate fit and adequate padding on pressure points, as per manufacturer's recommendations. Nursing guidelines for CS collar management included removal of the CS collar once per shift for skin assessment and washing of both skin and collar. CS collars are then reapplied and reassessed for fit. Patients in our PICU, unless contraindicated, were routinely positioned supine at a $30^{\circ}$ angle.

CS clearance at our institution followed published protocols for paediatric patients, ${ }^{13,14}$ but was before the Trauma Association of Canada consensus guidelines for evaluation of paediatric CS. ${ }^{15}$ Three views of the CS included an anterior-posterior view, lateral view, and odontoid view. Two additional oblique views were added at the discretion of the radiologist. Flexion/extension views were obtained for the investigation of potential subluxation or angulation of the cervical spine in relation to ligamentous laxity. Significant changes in CS clearance protocol over the study period consisted primarily of the addition of both CT/3D reconstruction and magnetic resonance imaging (MRI). Multi-slice CT scanning was used at our institution throughout the 10year study period, but dose reduction software was added.

All data were checked for normality. The mean and s.d. were used to describe normally distributed continuous variables, while the median and interquartile range was used for skewed continuous data. Student's $t$-test was used to compare mean differences in normally distributed continuous variables and skewed continuous variables were compared using the nonparametric Mann-Whitney $U$-test. Counts with percentages were used to summarize categorical variables. Differences in proportions between groups were compared using the Chi-square test. A $P<0.05$ was used to signify statistical significance. All analyses were performed using SPSS Version 14.0 (IBM Corporation, Armonk, NY, USA).

\section{RESULTS}

A total of 820 paediatric trauma patients over 10 years were screened. Of these, 365 patients were severely injured (ISS $\geqslant 12$ ). All were admitted to the PICU. The patient and injury demographics are presented in Table 1 . The predominant mechanism of injury was motor vehicle collisions, and the most common primary injury was TBI.

\section{Cervical spine injury}

Clinically significant CS injuries were identified in 5\% $(n=18 / 365)$ of trauma patients (Table 2). No CS injuries were identified in trauma patients with intentional injuries $(n=25)$, of which the vast majority were under 1 year of age, and diagnosed with shaken baby syndrome. In patients with CS injuries, motor vehicle collisions were the predominant mechanism of injury, and TBI was the primary injury. The incidence of CS injuries increased to $9 \%$ in severe TBI patients $(n=13 / 149)$. The majority of CS injury patients were ventilated (78\%; $n=14 / 18)$ on hospital admission and one-third of CS injury patients died $(33 \% ; n=6 / 18)$.

CS injuries were suspected before imaging in 33\% of patients ( $n=6 / 18)$ based on either motor/sensory impairment $(n=4 / 18)$ or shock $(n=2 / 18)$. CT scan was the first imaging method to identify CS injuries in $39 \%$ of patients $(n=7 / 18)$. One patient, who expired before CS imaging, had their CS injuries identified postmortem. MRI was used to confirm CS injuries in $67 \%(n=12 / 18)$ of patients. Of the six patients with CS injuries who did not receive MRI, two died before MRI could be completed, and one was transferred to another institution on PICU day 2. In the remaining three patients, it was unclear why an MRI was not done, however, flexion/extension views were taken in all cases.

All patients with CS injuries were over the age of 4 years. The mean age of patients with CS injuries was 11.8 years (s.d. $=3.7$ ), with $56 \%$ of injuries between C5-7 $(n=10 / 18)$. Removal of the rigid collar occurred in $39 \%(n=7 / 18)$ of these patients that had stable

\section{Table 1 Demographic and injury data for 365 severely injured} paediatric trauma patients

\begin{tabular}{lc}
\hline Mean age (years) & $10.1($ s.d. $=5.5)$ \\
Mean injury severity score & $28.1($ s.d. $=11.4)$ \\
Mean Glasgow Coma Scale & $9.2($ s.d. $=4.7)$ \\
Median paediatric intensive care unit stay (days) & $3($ IQR $=1-5)$ \\
Median hospital stay (days) & $10(I Q R=6-18)$
\end{tabular}

$\begin{array}{lc}\text { Mechanism of injury }(n(\%)) & \\ \text { Motor vehicle collision } & 139(38.1 \%) \\ \text { All-terrain vehicle/snowmobile } & 44(12.1 \%) \\ \text { Pedestrian vs. Car } & 39(10.7 \%) \\ \text { Bicycle injury } & 37(10.1 \%) \\ \text { Other } & 37(10.1 \%) \\ \text { Intentional injury } & 26(7.1 \%) \\ \text { Falls } & 25(6.8 \%) \\ \text { Farm injury } & 18(4.9 \%)\end{array}$

Primary injury ${ }^{\mathrm{a}}(\mathrm{n}(\%))$

Traumatic brain injury $\quad 172(49.1 \%)$

Multi-system $\quad 129(36.9 \%)$

Abdominal $\quad 36(10.3 \%)$

Thorax $9(2.6 \%)$

Spine $4(1.1 \%)$

Ventilated $(n(\%)) \quad 229(63 \%)$

Mortality $(n(\%)) \quad 56(15 \%)$

Abbreviation: IQR, interquartile range.

aFifteen patients were omitted from primary injuries due to inadequate documentation. 
Table 2 Clinically significant CS injuries identified in $5 \%(n=18 / 365)$ of severely injured paediatric trauma patients

\begin{tabular}{|c|c|c|c|c|c|c|}
\hline Age & Sex & $\begin{array}{l}\text { CS injury: } \\
\text { investigation }^{\text {a }}\end{array}$ & $\begin{array}{l}\text { CS injury: bone \# } \\
\text { and alignment }\end{array}$ & $\begin{array}{l}\text { CS injury: cord, ligament } \\
\text { and soft tissues }\end{array}$ & CS injury: Intervention & Outcome \\
\hline 5 & M & 3-films & C6-C7 distraction & Global cord oedema & CS collar & Brain dead \\
\hline 8 & $\mathrm{~F}$ & CT & $\begin{array}{l}\text { Atlanto-occipital dislocation } \\
\text { Atlanto-axial subluxation }\end{array}$ & Died before MR & CS collar & Brain dead \\
\hline 8 & $\mathrm{~F}$ & $\mathrm{~F} / \mathrm{E}$ & C2-C3 subluxation & normal & CS collar (4 weeks) & Alive \\
\hline 9 & M & CT & Odontoid \# (type 1) & Transferred before MR & CS collar on transfer & Alive \\
\hline 10 & M & 3-films & C2-C6 subluxation & normal & Stable & Alive \\
\hline 12 & M & CT & $\begin{array}{l}\text { C4-C5 wedge compressions } \\
\text { C5-C6 subluxation }\end{array}$ & $\begin{array}{l}\text { C3-C6 epidural hematoma } \\
\text { C3-C6 cord contusion } \\
\text { C4-C6 posterior ligament }\end{array}$ & C3-C6 fusion & $\begin{array}{l}\text { Alive } \\
\text { autonomic dysreflexia } \\
\text { parasthesia }\end{array}$ \\
\hline 12 & M & 3-films & $\begin{array}{l}\text { C3 inferior facet \# } \\
\text { C5 wedge \#, C6 burst \# }\end{array}$ & $\begin{array}{l}\text { C5-C6 oedema, hemorrhage } \\
\text { C5-C6 interspinous ligament disruption }\end{array}$ & C5-C7 fusion & $\begin{array}{l}\text { Alive } \\
\text { quadriplegia }\end{array}$ \\
\hline 14 & $\mathrm{~F}$ & 3-films & C7 spinous process & normal & Stable & Alive \\
\hline 15 & M & CT & $\mathrm{C} 2$ rotation/subluxation & normal & Stable & Alive \\
\hline 15 & M & MRI & normal & $\mathrm{C} 5 / 6$ and $\mathrm{C} 6 / 7$ disk herniations & Stable & Alive \\
\hline 17 & $\mathrm{~F}$ & СТ & C7-T1 transverse process \# & normal & Stable & Alive \\
\hline 17 & $\mathrm{~F}$ & CT & C7 spinous process \# & normal & Stable & Brain dead \\
\hline 17 & M & СT & $\begin{array}{l}\text { C5-C7 lamina \# } \\
\text { C6-C7 vertebral body \# }\end{array}$ & $\begin{array}{l}\text { C5C7 disk herniation posteriorly } \\
\text { C6-C7 cord compression/contusion }\end{array}$ & C2-C5 fusion & $\begin{array}{l}\text { Alive } \\
\text { quadriplegia }\end{array}$ \\
\hline
\end{tabular}

Abbreviations: CS, cervical spine; CT, computed tomography; MRI, magnetic resonance imaging. aFirst CS investigation to identify clinically significant injury.

Table 3 Subgroup analyses for severely injured paediatric trauma patients with and without CS injuries

\begin{tabular}{lccc}
\hline & $\begin{array}{c}\text { CS injury } \\
(\mathrm{n}=18)\end{array}$ & $\begin{array}{c}\text { No CS injurya } \\
(\mathrm{n}=322)\end{array}$ & P-value \\
\hline Age (years), mean (s.d.) & $11.8(3.7)$ & $10.6(5.1)$ & 0.22 \\
Male, \% & 55.6 & 65.2 & 0.40 \\
Traumatic brain injury, \% & 72.2 & 66.1 & 0.60 \\
Injury severity score, mean (s.d.) & $38.4(20.2)$ & $27.8(10.8)$ & 0.04 \\
Glasgow coma scale, mean (s.d.) & $9.2(5.1)$ & $9.4(4.6)$ & 0.88 \\
Paediatric intensive care unit days, & $4.0(2.0-8.5)$ & $2.0(1.0-4.0)$ & 0.02 \\
median (IQR) & & & \\
Hospital days, median (IQR) & $16.5(9.5-34.0)$ & $10.0(6.0-17.0)$ & 0.05 \\
Days to CS clearance, median (IQR) & $5.5(3.5-15.0)$ & $2.0(1.0-5.0)$ & 0.02 \\
\hline
\end{tabular}

Abbreviations: CS, cervical spine; IQR, interquartile range.

antentional injuries were excluded $(n=25)$.

CS injuries. Patients with CS injuries had significantly higher ISS, longer PICU and hospital stays, and increased times to CS collar removal, with or without CS stabilization (Table 3).

\section{Cervical spine collar complications}

CS collar complications occurred in $10 \%$ of patients $(n=35 / 365)$, consisting of either erythema $(46 \% ; n=16 / 35)$ or pressure sores (54\%; $n=19 / 35)$. Only one child with CS collar complications had a CS injury identified. Most patients with CS collar complications were ventilated (97\%; $n=34 / 35)$ and had TBI $(83 \% ; n=29 / 35)$. Intracranial pressure monitors were placed in $31 \%$ of patients with CS collar complications $(n=11 / 35)$. CS collar complications were often identified between PICU days 3-7 (77\%; $n=27 / 35)$, with the peak number of CS collar complications identified on day 6. Patients with CS collar complications were older, had a lower GCS, had longer PICU and hospital stays and had increased days to CS clearance (Table 4). Lesions caused by the CS collar were typically located on the chin, neck and shoulders. Only four charts documented the type of collar the patient was wearing when lesions were discovered (3 Philadelphia, 1 hard collar).

\section{Cervical spine clearance}

The median time to CS clearance was 2 days for those without CS injuries or CS collar complications, 5.5 days for the seven patients with stable CS injuries, and 7 days for those with CS collar complications (Figure 1a, Tables 3 and 4). Those CSs cleared on day 1-2 were significantly more likely to be cleared using clinical exam, and less likely to be cleared by CT/MRI or CT with flexion/ extension views (Table 5). CS were cleared in $26 \%$ of patients with a combination of both clinical exam and imaging (three films or CT; $n=70)$, with $13 \%(n=36)$ of CSs cleared by clinical exam only. The primary method of clearance was three films with flexion/extension views $(31 \% ; n=84$; Figure $1 \mathrm{~b})$. Those patients who had their CS cleared with CT/MRI were more likely to be over the age of 8 years, have a TBI and/or a GCS $\leqslant 8$ and most were not cleared until after hospital day 2 (Table 5 ). 
Table 4 Subgroup analyses for severely injured paediatric trauma patients with and without CS collar complications

\begin{tabular}{lccc}
\hline & $\begin{array}{c}\text { CS collar } \\
\text { complications } \\
(\mathrm{n}=35)\end{array}$ & $\begin{array}{c}\text { No CS collar } \\
\text { complications }\end{array}$ & P-value \\
& $(\mathrm{n}=305)$ & \\
\hline Age (years), mean (s.d.) & $12.2(4.1)$ & $10.5(5.1)$ & 0.03 \\
Male, \% & 57.1 & 65.6 & 0.32 \\
Traumatic brain injury, \% & 82.9 & 64.6 & 0.03 \\
Injury severity score, mean (s.d.) & $29.8(10.0)$ & $28.2(11.9)$ & 0.43 \\
Glasgow Coma Scale, mean (s.d.) & $6.9(3.6)$ & $9.7(4.7)$ & $<0.001$ \\
Paediatric intensive care unit days, & $6.0(4.0-10.0)$ & $2.0(1.0-4.0)$ & $<0.001$ \\
median (IQR) & & & \\
Hospital days, median (IQR) & $20.5(11.8-38.3)$ & $9.0(5.0-15.0)$ & $<0.001$ \\
Days to CS clearance, median & $7.0(5.0-11.0)$ & $2.0(1.0-4.0)$ & $<0.001$ \\
(IQR) & & & \\
\hline Abbreviations: CS, cerval & & &
\end{tabular}

Abbreviations: CS, cervical spine; IQR, interquartile range.

alntentional inures were excluded $(n=25)$.
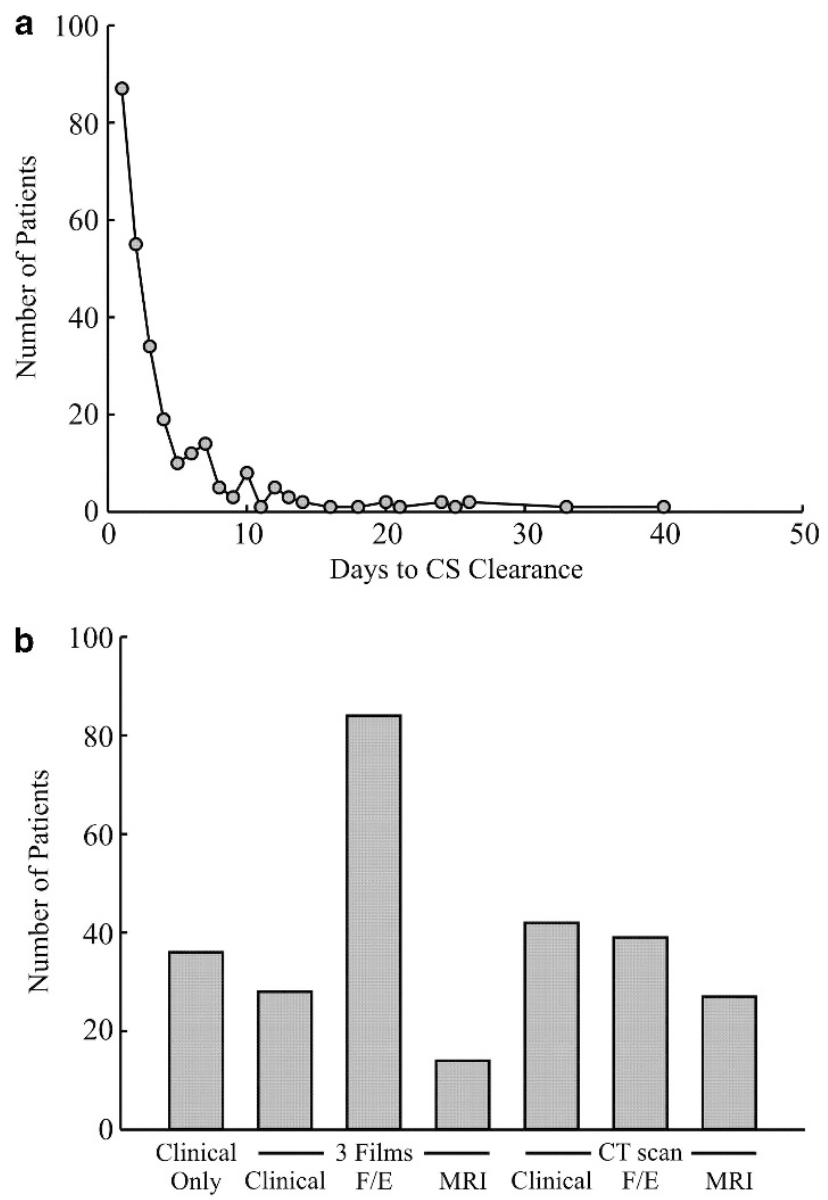

CS Clearance Investigations

Figure $1 \mathrm{CS}$ clearance and CS collar complications in severely injured paediatric trauma patients admitted to the critical care unit. (a) A plot reflecting the number of patients whom had their CS cleared relative to the day of hospitalization. The median time to clearance was 2.0 days. (b) A plot reflecting CS clearance investigations for each trauma patient. The majority of CS were cleared using with three films and flexion/extension views.

\section{DISCUSSION}

Paediatric CS injuries are potentially devastating, often resulting in severe morbidity and mortality. To our knowledge this study is one of the few to investigate CS injuries, and to report CS collar complications, in paediatric severe trauma patients. The demographic and injury data presented herein are consistent with previous paediatric trauma studies, ${ }^{5,16-18}$ and are therefore, likely representative of severe trauma patients in most paediatric trauma hospitals.

The first key finding of our study was that the incidence of CS injuries in our severely injured trauma population was 5\%; significantly higher than previously reported in the general trauma population $(0.2-1.6 \%){ }^{1-5}$ The higher incidence of CS injuries was likely due to the greater severity of injuries and larger proportion of motor vehicle collisions. ${ }^{2}$ It is also possible that some CS injuries were missed in earlier reports. Importantly, the incidence of CS injuries increased to $9 \%$ in association with TBI and $11 \%$ in trauma deaths. Most CS injured patients were ventilated and had TBI, resulting in delayed collar removal and longer hospital stays. A trend was evident between the age and level of CS injury, whereby older patients generally had lower CS injuries occurring in C5-C7. Children over the age of 8 years have anatomy similar to adults, and thus the most common injury location in these children is in the lower CS. ${ }^{3,4,14}$

A second key finding of our study was that CS collar complications occurred in $10 \%$ of our severely injured trauma patients; consisting of either erythema or ulcers. CS collar complications were associated with longer times to CS clearance, lower GCS scores and longer PICU and hospital stays. The prevalence of CS collar complications in adults is reported in the range of $7-23 \%,{ }^{9,10}$ and CS collar complications correlate with higher ISS and increased days of ventilation and intracranial pressure monitoring. ${ }^{10}$

The median day for CS clearance was 2 days for those patients who had neither CS injuries nor collar complications. A fast CS clearance time is imperative and clinical CS clearance is ideal, but our rates were relatively low at $13 \%$ (adults 20\%), ${ }^{13}$ likely due to the higher severity of injuries in our trauma population. Indeed, clinical assessment is less reliable for patients with added comorbidities, lower GCSs and distracting injuries. Clinical CS clearance requires that patients have no midline cervical tenderness, no intoxication, no pain and no neurological deficits. ${ }^{19}$

At our centre, CS clearance follows published protocols for paediatric patients, ${ }^{13,14}$ but was before publication of the Trauma Association of Canada consensus guidelines for CS evaluation. ${ }^{15}$ Overall, three CS films with flexion/extension views were used most often to clear CSs in trauma patients. More recently, CS clearance relied increasingly on CT/MRI, primarily in patients with a low GCS or TBI, consistent with previous research, which suggests MRI to be beneficial in CS clearance for those paediatric patients who are obtunded, ventilated or unco-operative. ${ }^{6}$ Although studies suggest that ligamentous injuries in obtunded paediatric patients are accurately identified with MRI, ${ }^{6}$ the optimal timing for accurate identification of CS injuries is debatable. ${ }^{20}$ MRI has been shown to be an effective tool to rule out ligamentous and soft tissue injury owing to its sensitivity for patients who present with spinal cord injury without radiological abnormality, a phenomenon seen almost exclusively in children. ${ }^{6}$

Multidisciplinary approaches to decrease CS collar complications have been successful. Emphasis on optimal CS collar management includes: removing the hard collar and changing to a more long-term collar in the first $24 \mathrm{~h}$ following presentation to hospital; making certain that the collar is properly fit and that there are 
Table 5 A comparison of patient, injury and outcome variables by CS clearance methods in severely injured paediatric trauma patients $(n=270)$

\begin{tabular}{|c|c|c|c|c|c|c|c|c|}
\hline & \multicolumn{2}{|c|}{ Age (years) } & \multicolumn{2}{|c|}{ Glasgow coma scale } & \multicolumn{2}{|c|}{ Traumatic brain injury } & \multicolumn{2}{|c|}{ CS clearance day } \\
\hline & $\leqslant 8$ years $(n=91)$ & $>8$ years $(n=179)$ & $\leqslant 8(n=114)$ & $>8(n=156)$ & $Y(n=172)$ & $N(\mathrm{n}=98)$ & $\leqslant 2(n=142)$ & $>2(n=128)$ \\
\hline Clinical only & 12 & 24 & 3 & $33^{c}$ & 8 & $28^{c}$ & 34 & $2^{d}$ \\
\hline 3 films + clinical & 13 & 15 & 5 & $23^{a}$ & 17 & 11 & 21 & 7 \\
\hline 3 films + flexion/extension & 25 & 59 & 43 & 41 & 59 & 25 & 41 & 43 \\
\hline 3 films + MRI & 7 & 7 & 9 & 5 & 13 & $1^{\mathrm{a}}$ & 5 & 9 \\
\hline CT+clinical & 17 & 25 & 12 & 30 & 22 & 20 & 27 & 15 \\
\hline $\mathrm{CT}+$ flexion/extesion & 13 & 26 & 22 & 17 & 28 & 11 & 10 & $29^{c}$ \\
\hline $\mathrm{CT}+\mathrm{MRI}$ & 4 & $23^{a}$ & 20 & $7^{b}$ & 25 & $2^{b}$ & 4 & $23^{d}$ \\
\hline
\end{tabular}

Abbreviations: CS, cervical spine; CT, computed tomography; MRI, magnetic resonance imaging.

Seventy patients were excluded from analyses due to either insufficient chart data or death prior to CS clearance. All data groups compared with Chi-square test.

Seventy patien
a $P<0.05$.

${ }^{\mathrm{b}} P<0.01$.

${ }^{\mathrm{c}} P<0.001$.

${ }^{\mathrm{d}} P<0.0001$.

no areas of increased pressure, which can be avoided; ensuring the skin is inspected and cared for at least every $8 \mathrm{~h}$; using foam or other padding to cushion potential pressure points; and documenting skin conditions every nursing shift. ${ }^{2}$ An additional way to decrease CS collar complications is to select a collar, which decreases moisture and skin pressure, such as Aspen and Miami collars. ${ }^{11}$ CS collar management is imperative in paediatric trauma patients, who present with a low GCS, as these patients experience longer CS clearance times and a greater number of PICU and hospital days.

Our study has several limitations. First, retrospective studies have the potential for missed data. Second, CS injuries may be underestimated as some patients were not imaged with both CT and MRI. The rates of CT/MRI did increase over the 10-year study period. Third, CS injuries were identified only in children greater than 5 years of age and thus, our results may not be applicable to younger patients. Lastly, those patients who died before hospital admission would not have been captured.

In summary, we report several important findings with regards to severely injury patients that are primarily admitted to the PICU. First, we showed a significantly higher CS injury rate in severely injured paediatric trauma patients $(5 \%)$ as compared with the general trauma population $(0.2-1.6 \%){ }^{1-5}$ Further study is required to verify that differences in the mechanisms of injury (that is, motor vehicle collisions versus falls from height $)^{2}$ underlied the increased incidence of CS injuries, rather than missed injuries in previous reports. Second, our study revealed a significant rate of CS collar complications (10\%), consisting of either erythema or ulcerations. Attention to CS collar management, particularly in ventilated patients with lower GCS, in combination with rapid CS clearance with CT/ MRI will likely prevent CS collar complications.

\section{DATA ARCHIVING}

There were no data to deposit.

\section{CONFLICT OF INTEREST}

The authors declare no conflict of interest.
1 Viccellio P, Simon H, Pressman BD, Shah MN, Mower WR, Hoffman JR. A prospective multicenter study of cervical spine injury in children. Pediatrics 2001; 108, E20.

2 Hofbauer M, Jaindl M, Hochtl LL, Ostermann RC, Kdolsky R, Aldrian S. Spine injuries in polytraumatized pediatric patients: characteristics and experience from a Level I trauma center over two decades. J Trauma Acute Care Surg 2012; 73, 156-161.

3 Mohseni S, Talving P, Branco BC, Chan LS, Lustenberger T, Inaba K et al. Effect of age on cervical spine injury in pediatric population: a National Trauma Data Bank review. J pediatr surg 2011; 46, 1771-1776.

4 Kokoska ER, Keller MS, Rallo MC, Weber TR. Characteristics of pediatric cervical spine injuries. J pediatr surg 2001; 36, 100-105.

5 Patel JC, Tepas JJ 3rd, Mollitt DL, Pieper P. Pediatric cervical spine injuries: defining the disease. J pediatr surg 2001; 36, 373-376.

6 Frank JB. The efficacy of MRI in pediatric cervical spine clearance. Spine 2002; 27 1176-1179.

7 Lustrin ES, Karakas SP, Ortiz AO, Cinnamon J, Castillo M, Vaheesan $\mathrm{K}$ et al. Pediatric cervical spine: normal anatomy, variants, and trauma. Radiographics 2003; 23, 539-560.

8 Pizzutillo PD. Injury of the cervical spine in young children. Instr Course Lect 2006; $55,633-639$.

9 Powers J, Daniels D, McGuire C, Hilbish C. The incidence of skin breakdown associated with use of cervical collars. J Trauma Nurs 2006; 13, 198-200.

10 Molano Alvarez E, Murillo Perez Mdel A, Salobral Villegas MT, Dominguez Caballero M, Cuenca Solanas M, Garcia Fuentes C. [Pressure sores secondary to immobilization with cervical collar: a complication of acute cervical injury]. Enferm Intensiva 2004; 15, 112-122.

11 Plaisier B, Gabram SG, Schwartz RJ, Jacobs LM. Prospective evaluation of craniofacial pressure in four different cervical orthoses. J trauma 1994; 37, 714-720.

12 Powers J. A multidisciplinary approach to occipital pressure ulcers related to cervical collars. J Nurs Care Qual 1997; 12, 46-52.

13 Browne GJ, Lam LT, Barker RA. The usefulness of a modified adult protocol for the clearance of paediatric cervical spine injury in the emergency department. Emerg Med (Fremantle) 2003; 15, 133-142.

14 Platzer P, Jaind M, Thalhammer G, Dittrich S, Kutscha-Lissberg F, Vecsei V et al. Cervical spine injuries in pediatric patients. J trauma 2007; 62, 389-396. discussion 394-6.

15 Chung S, Mikrogianakis A, Wales PW, Dirks P, Shroff M, Singhal A et al. Trauma association of Canada Pediatric Subcommittee National Pediatric Cervical Spine Evaluation Pathway: consensus guidelines. J trauma 2011; 70, 873-884.

16 Givens TG, Polley KA, Smith GF, Hardin WD Jr. Pediatric cervical spine injury: a threeyear experience. J trauma 1996; 41, 310-314.

17 Duhem R, Tonnelle V, Vinchon M, Assaker R, Dhellemmes P. Unstable upper pediatric cervical spine injuries: report of 28 cases and review of the literature. Childs Nerv Syst 2008; 24, 343-348.

18 Polk-Williams A, Carr BG, Blinman TA, Masiakos PT, Wiebe DJ, Nance ML. Cervical spine injury in young children: a National Trauma Data Bank review. J Pediatr Surg 2008; 43, 1718-1721.

19 Hoffman JR, Mower WR, Wolfson AB, Todd KH, Zucker ML. Validity of a set of clinical criteria to rule out injury to the cervical spine in patients with blunt trauma. National Emergency X-Radiography Utilization Study Group. N Eng/ J Med 2000; 343, 94-99.

20 Hadley MN, Walters BC, Grabb PA, Oyesiku NM, Przybylski GJ, Resnick DK et al. Guidelines for the management of acute cervical spine and spinal cord injuries. Clin Neurosurg 2002; 49, 407-498. 